\title{
A QUADRATIC FORM PROBLEM IN THE CALCULUS OF VARIATIONS*
}

\section{A. ADRIAN ALBERT}

The problem which we shall discuss arose in connection with sufficiency theorems in the multiple integral problem of the calculus of variations. It was proposed by Professor G. A. Bliss to his University of Chicago seminar (summer, 1937) and communicated to the author by Professor W. T. Reid. The result of the author's investigation presented here is a very interesting theorem on real quadratic forms.

We first have the trivial lemma:

LEMMA 1. Let $f$ and $g$ be real quadratic forms in $x_{1}, \cdots, x_{n}$, and $g$ be negative definite. Then there exists a real non-singular linear transformation carrying $g$ and $f$ respectively into

$$
G=-\left(x_{1}^{2}+\cdots+x_{n}^{2}\right), \quad F=\lambda_{1} x_{1}^{2}+\cdots+\lambda_{n} x_{n}^{2},
$$

where the $\lambda_{i}$ are the roots of the determinant $|f+\lambda g|=0 \dagger$ and may be arranged so that

$$
\lambda_{n} \geqq \lambda_{n-1} \geqq \cdots \geqq \lambda_{1} .
$$

Moreover $f+\lambda g$ is positive definite if and only if

$$
\lambda_{1}>\lambda>-\infty \text {. }
$$

For we may carry $g$ into $G$. Apply a real orthogonal transformation carrying the resulting $f$ into diagonal form $F$. The $\lambda_{i}$ are clearly the roots of $|F+\lambda G|=0$ and hence of $|f+\lambda g|=0$. Finally $f+\lambda g$ is positive definite if and only if $F+\lambda G$ is positive definite, that is if $\lambda_{i}-\lambda \geqq \lambda_{1}-\lambda>0$.

We next derive the following lemma:

Lemma 2. Let $g$ be non-singular and indefinite of index $p$, and let there exist a real $\lambda_{0}$ such that $f+\lambda_{0} g=h$ is positive definite. Then there exists a real non-singular linear transformation carrying $g$ and $f$ respectively into

$$
\begin{aligned}
& G=x_{1}^{2}+\cdots+x_{p}^{2}-\left(x_{p+1}^{2}+\cdots+x_{n}^{2}\right), \\
& F=-\left(\lambda_{1} x_{1}^{2}+\cdots+\lambda_{p} x_{p}^{2}\right)+\lambda_{p+1} x_{p+1}^{2}+\cdots+\lambda_{n} x_{n}^{2},
\end{aligned}
$$

\footnotetext{
* Presented to the Society, November 27, 1937.

$\dagger$ By this we mean the determinant of the matrix corresponding to the pencil of forms $f+\lambda g$.
} 
with $\lambda_{i}$ the roots of $|f+\lambda g|=0$. Moreover

$$
\lambda_{j}>\lambda_{i}, \quad i=1, \cdots, p ; j=p+1, \cdots n,
$$

so that we may arrange the roots in the order

$$
\lambda_{n} \geqq \lambda_{n-1} \geqq \cdots \geqq \lambda_{p+1}>\lambda_{p} \geqq \lambda_{p-1} \geqq \cdots \geqq \lambda_{1} .
$$

Finally $f+\lambda g$ is positive definite if and only if

$$
\lambda_{p+1}>\lambda>\lambda_{p}
$$

For as in Lemma 1 we carry $h$ to $\xi_{1}^{2}+\cdots+\xi_{n}^{2}$ and then apply an orthogonal transformation leaving $h$ unaltered and carrying $g$ into $\sum_{i=1}^{n} \delta_{i} \xi_{i}^{2}$ for real $\delta_{i} \neq 0$. Let $x_{i}=\left|\delta_{i}\right|^{1 / 2} \xi_{i}$; then $g=\sum_{i=1}^{n} \pm x_{i}^{2}$, $h=\sum_{i=1}^{n}\left|\delta_{i}\right|^{-1} x_{i}{ }^{2}$. The index of $g$ is an invariant and $g$ may then be carried into $G$. But $h$ is diagonal and hence so is $f=h-\lambda_{0} g$. Write $f=F$ as in (3); then $F+\lambda G=\sum_{i=1}^{p}\left(\lambda-\lambda_{i}\right) x_{i}{ }^{2}+\sum_{j=p+1}^{n}\left(\lambda_{j}-\lambda\right) x_{j}{ }^{2}$ so that the $\lambda_{i}$ are the characteristic roots of $|f+\lambda g|=0$. The value $x_{k}=0$ for $k \neq i$ and $j, x_{i}=x_{j}=1, i$ and $j$ as in (4), gives $g=0$, $h=f+\lambda_{0} g=f=\lambda_{j}-\lambda_{i}>0$, and we have (4). Then $F+\lambda G$, and hence $f+\lambda g$, is positive definite if and only if $\lambda_{j}>\lambda>\lambda_{i}$, which is satisfied if and only if (6) holds.

Observe that Lemma 1 becomes a special case of Lemma 2 if we allow $p=0$ and $\lambda_{0}=-\infty$.

Theorem. Let $f$ and $g$ be real quadratic forms in $x_{1}, \cdots, x_{n}$, and let $f$ be positive for all real $x_{i}$ not all zero such that $g=0$. Then there exists a real number $\lambda$ such that $f+\lambda g$ is positive definite.

The properties of the theorem are clearly invariant under real non-singular linear transformations, and under replacement of $g$ by $\mu g$ for $\mu$ real and not zero. We shall use such transformations.

The result is true for $n=1$ since then either $g=0$ and $f$ is necessarily positive definite, or we may take $g=x^{2}, f=a x^{2}, f+(1-a) g=x^{2}$ positive definite. We thus make an induction and assume our theorem for forms in $n-1$ variables.

If the rank of $g$ were $r<n$, we could take $g$ to be a form in $x_{1}, \cdots, x_{r}$. Then $x_{1}=\cdots=x_{r}=0$ in $f$ gives a form $f_{2}=f_{2}\left(x_{r+1}, \cdots, x_{n}\right)>0$ for all $x_{r+1}, \cdots, x_{n}$ not all zero. Hence $f_{2}$ is definite and may be taken to be $x_{r+1}^{2}+\cdots+x_{n}^{2}$ by a transformation on $x_{r+1}, \cdots, x_{n}$ not altering $g$. Thus $f=x_{r+1}^{2}+\cdots+x_{n}{ }^{2}+2 \sum_{t=r+1}^{n} x_{i} L_{i}+f_{1}\left(x_{1}, \cdots, x_{r}\right)$, where the $L_{i}$ are linear forms in $x_{1}, \cdots, x_{r}$. The transformation

$$
X_{i}=x_{i}+L_{i}, \quad X_{j}=x_{j}, \quad j=1, \cdots, r ; i=r+1, \cdots, n,
$$


does not alter the form of $g$ and carries $f$ into $X_{r+1}^{2}+\cdots+X_{n}^{2}$ $+F\left(X_{1}, \cdots, X_{r}\right), g$ into $G\left(X_{1}, \cdots, X_{r}\right)$. Put $X_{r+1}=\cdots=X_{n}=0$ and have $F>0$ for all $X_{j}$ not all zero such that $G=0$. Then $F+\lambda G$ is positive definite for a real $\lambda$ and so is $X_{r+1}^{2}+\cdots+X_{n}^{2}+F+\lambda G=f+\lambda g$.

Hence let $g$ be non-singular. Our result follows from Lemma 1 if $g$ is definite. Now let $g$ be indefinite and apply a linear transformation carrying $g$ into the form (3). Then $f_{0}=f\left(0, x_{2}, \cdots, x_{n}\right)>0$ for all $x_{2}, \cdots, x_{n}$ not all zero and making $g_{0}=g\left(0, x_{2}, \cdots, x_{n}\right)=0$. By the hypothesis of our induction we may apply Lemma 2 and write either

$$
f_{0}=-\left(\lambda_{2} x_{2}^{2}+\cdots+\lambda_{p} x_{p}^{2}\right)+\lambda_{p+1} x_{p+1}^{2}+\cdots+\lambda_{n} x_{n}^{2},
$$

where

$$
\lambda_{n} \geqq \lambda_{n-1} \geqq \cdots \geqq \lambda_{p+1}>\lambda_{p} \geqq \lambda_{p-1} \geqq \cdots \geqq \lambda_{2},
$$

or

$$
p=1, \quad f_{0}=\lambda_{2} x_{2}^{2}+\cdots+\lambda_{n} x_{n}^{2}, \quad \lambda_{n} \geqq \cdots \geqq \lambda_{2} .
$$

In the respective cases we take $\lambda$ in the intervals

$$
\lambda_{p+1}>\lambda>\lambda_{p}, \quad \lambda_{2}>\lambda>-\infty .
$$

Now

$$
f=f_{0}+2 \sum_{i=2}^{n} b_{i} x_{i} x_{1}+a x_{1}^{2}
$$

so that

$$
\begin{aligned}
f+\lambda g= & \sum_{i=2}^{p}\left(\lambda-\lambda_{i}\right)\left(x_{i}+\frac{b_{i} x_{1}}{\lambda-\lambda_{i}}\right)^{2} \\
& +\sum_{j=p+1}^{n}\left(\lambda_{j}-\lambda\right)\left(x_{j}+\frac{b_{j} x_{1}}{\lambda_{j}-\lambda}\right)^{2}+x_{1}^{2} \phi(\lambda),
\end{aligned}
$$

where

$$
\phi(\lambda)=a+\lambda-\left(\sum_{i=2}^{p} \frac{b_{i}{ }^{2}}{\lambda-\lambda_{i}}+\sum_{j=p+1}^{n} \frac{b_{j}^{2}}{\lambda_{j}-\lambda}\right) .
$$

The values

$$
\begin{array}{r}
x_{i}=-\frac{b_{i}}{\lambda-\lambda_{i}}, \quad x_{j}=-\frac{b_{j}}{\lambda_{j}-\lambda}, \quad x_{1}=1, \\
i=2, \cdots, p ; j=p+1, \cdots, n,
\end{array}
$$

make 


$$
f+\lambda g=\phi(\lambda), g=1+\sum_{i=2}^{p} \frac{b_{i}{ }^{2}}{\left(\lambda-\lambda_{i}\right)^{2}}-\sum_{j=p+1}^{n} \frac{b_{j}^{2}}{\left(\lambda_{j}-\lambda\right)^{2}}=\phi^{\prime}(\lambda),
$$

a rational function of $\lambda$ continuous in the interval (11).

If all the $b_{k} \neq 0$ we have $\phi^{\prime}\left(\lambda_{p+1}\right)=-\infty, \phi^{\prime}\left(\lambda_{p}\right)=\infty$ if $p>1$, while if $p=1$, then $\phi^{\prime}(-\infty)=1>0$. Hence there exists a $\lambda$ in the intervals (10) such that $\phi^{\prime}(\lambda)=g=0$. But then our hypothesis states that $f=\phi(\lambda)>0$. By (12), and since $\phi(\lambda)>0$, we have $f+\lambda g$ positive definite.

There remains the case where some $b_{k}=0$. Here we may permute the $x_{i}$ and change the sign of $g$ if necessary and carry the corresponding $x_{k}$ into $x_{1}$. Then $f=-\lambda_{1} x_{1}^{2}+f_{0}\left(x_{2}, \cdots, x_{n}\right)$. As in the proof above we may carry $f_{0}$ into (7) and have $f$ in the form (3). But $f>0$ for $g=0$ and as in the proof of Lemma 2 we have (5), and $f+\lambda g$ is positive definite for $\lambda$ as in (6).

We have proved our theorem. Notice that our reduction to the case $g$ non-singular together with Lemmas 1,2 determines the range of $\lambda$ for which $f+\lambda g$ is positive definite.

The University of Chicago

\section{THE RIEMANNIAN CURVATURE OF A HYPERSURFACE*}

\section{AARON FIALKOW $\dagger$}

1. Introduction. It is a well known theorem of Gauss that the total curvature of any two dimensional surface in euclidean three space is equal to the product of the principal normal curvatures. Eisenhart $\ddagger$ has shown that a generalization of this theorem applies to Riemann spaces of class one; that is, the hypersurfaces of an $n$-dimensional flat space. He proves the theorem:

When the lines of curvature of a Riemann space $V_{n}$ of class one are real and none of them is tangent to a null vector, the Riemannian curvature at a point for the orientation determined by the direction of two lines of curvature at the point is numerically equal to the product of the corresponding normal curvatures; the sign is determined by the character of the normal to $V_{n}$ in the enveloping flat $V_{n+1}$.

* Presented to the Society, September 10, 1937.

$\dagger$ National Research Fellow.

$\ddagger$ L. P. Eisenhart, Riemannian Geometry, 1926, p. 199. 